\title{
Análise Descritiva do Perfil dos Alunos do Bacharelado em Física Médica e em Física da Universidade Federal do Rio de Janeiro
}

Descriptive profile analysis of undergraduate students of Medical Physics and Physics at Federal University of Rio de Janeiro

\author{
Bruna Lamis Alvarenga ${ }^{* 1,2 @, ~ R o s i m a r y ~ T e r e z i n h a ~ d e ~ A l m e i d a ~}{ }^{2 @}$, Odair Dias Gonçalves ${ }^{2}$ \\ ${ }^{1}$ Instituto de Radioproteção e Dosimetria, Rio de Janeiro, RJ, Brasil \\ ${ }^{2}$ Universidade Federal do Rio de Janeiro, Instituto de Física, Rio de Janeiro, RJ, Brasil \\ ${ }^{3}$ Instituto Alberto Luiz Coimbra de Pós-Graduação e Pesquisa de Engenharia (COPPE), Programa de Engenharia \\ Biomédica, Rio de Janeiro, RJ, Brasil
}

Recebido em 31 de julho de 2020. Revisado em 16 de setembro de 2020. Aceito em 22 de setembro de 2020.

Este trabalho tem o objetivo de analisar o perfil dos alunos dos cursos de Bacharelado em Física e em Física Médica do Instituto de Física da Universidade Federal do Rio de Janeiro. O período analisado foi de 2011 até 2018, em função de variáveis como: sexo, cor, notas das provas de seleção (ENEM) para entrar na universidade, categoria de entrada (ampla concorrência ou cota), Coeficiente de Rendimento Acumulado (CRA) e notas em Física I, Física II, Cálculo I e Cálculo II. As médias das notas do ENEM para acesso ao curso são diferentes entre dos alunos de Física e de Física Médica (valor p $<2,20 \mathrm{e}-16$ ), bem como as médias dos CRAs dos alunos ativos dos dois cursos (valor $\mathrm{p}=7,47 \mathrm{e}-8$ ). No entanto, essas diferenças desaparecem entre os alunos que concluíram o curso (valor $\mathrm{p}=0,38$ ). Cálculo I e Física I são as disciplinas nas quais os alunos de Física Médica têm desempenho mais baixo, podendo ser um dos fatores que contribuem para a diferença das médias de CRA entre os alunos ativos dos dois cursos. Essa diferença desaparece em Cálculo II e Física II.

Palavras-chave: física médica, bacharelado em física, desempenho acadêmico, ENEM, cotas.

This work aims to evaluate the profile of undergraduate students of Physics and Medical Physics courses at the Institute of Physics of the Federal University of Rio de Janeiro, Brazil. The period analyzed was from 2011 to 2018, due to variables such as: sex, color, university entrance exam score (ENEM), entry category (traditional or quota), cumulated academic performance coefficient (CRA) and grades in Physics and Calculus basic courses. The average ENEM scores are different among Physics and Medical Physics students ( $\mathrm{p}$ value $<2.20 \mathrm{e}-16$ ), as well as the average CRA scores of active students ( $p$ value $=7.47 \mathrm{e}-8$ ). However, these differences disappear between students who completed the course $(\mathrm{p}$ value $=0.38$. Calculus I and Physics I are the subjects in which Medical Physics students perform poorly, which partly justifies the difference in CRA between active students. The difference is not significant for Calculus II and Physics II.

Keywords: Medical Physics, Physics; academic performance, university entrance exam, social quotas.

\section{Introdução}

O curso de Física Médica do Instituto de Física da Universidade Federal do Rio de Janeiro (UFRJ) foi criado em 1998 como uma Habilitação do curso de Bacharelado em Física. Foi aprovado pelo Conselho de Ensino de Graduação (CEG) em 2000 e reconhecido pelo Ministério da Educação (MEC) em 2003. A finalidade de sua criação foi atender à demanda por Físicos Médicos na área hospitalar em função do crescente número de equipamentos que fazem uso da radiação ionizante na assistência à saúde. Esses profissionais têm suas atividades descritas na norma CNEN NN 6.10 para a Radioterapia, na norma CNEN NN 3.05 para a Medicina

\footnotetext{
* Endereço de correspondência: bruna.lamis@hotmail.com
}

Nuclear e na obsoleta Portaria 453 da Agência Nacional de Vigilância Sanitária (ANVISA), revogada pela Resolução da Diretoria Colegiada RDC 330, para o Radiodiagnóstico [1-4].

Como o curso é de natureza interdisciplinar, para a sua criação, o Instituto de Física (IF) contou com a colaboração do Instituto de Matemática (IM), do Instituto de Biofísica Carlos Chagas Filho (IBCCF), do Instituto de Bioquímica Médica (IBqM), do Instituto de Radioproteção e Dosimetria (IRD), do Instituto de Engenharia Nuclear (IEN), do Instituto Alberto Luiz Coimbra de Pós-Graduação e Pesquisa em Engenharia (COPPE), do Hospital Universitário Clementino Fraga Filho (HUCFF) e do Instituto Nacional do Câncer (INCA).

Em 2009, a coordenação do curso propôs a alteração de Habilitação para Bacharelado independente, sendo 
aprovada pelo CEG em 2010 e reconhecida pelo MEC em 2012. Portanto, 2011 foi o primeiro ano do bacharelado em Física Médica.

Os dois primeiros anos (ciclo básico) do bacharelado em Física e em Física Médica são parcialmente similares, tendo 13 disciplinas em comum, dentre essas: cálculos, físicas básicas, físicas experimentais e métodos computacionais. Os outros dois anos (ciclo profissional) dos cursos tinham apenas uma disciplina em comum: Termodinâmica Física Estatística, porém, na nova grade de Física Médica (estabelecida em 2019), essa disciplina se tornou eletiva [5].

Desde 2012, a entrada dos alunos nos cursos ocorre via o Exame Nacional do Ensino Médio (ENEM). Nesse mesmo ano, entrou em vigor a lei $\mathrm{n}^{\circ} 12.711$, chamada Lei das Cotas, que determina que $50 \%$ das vagas da instituição devem ser destinadas aos alunos que cursaram integralmente o ensino médio em escolas públicas. Essa lei foi implantada integralmente na UFRJ em 2014 [6].

Passados 20 anos, o curso de Física Médica ainda apresenta uma taxa de desistência mais alta do que a esperada pela coordenação. Diante desse quadro, torna-se importante analisar o desempenho (coeficiente de rendimento acumulado e notas nas disciplinas do ciclo básico) dos alunos dos dois cursos, bem como suas características demográficas (sexo, cor), categoria de entrada e nota na seleção para ingressar no curso.

O objetivo deste trabalho é descrever e analisar o perfil dos alunos dos dois cursos, disponibilizando informação no sentido de orientar políticas de apoio pedagógico aos alunos com maior defasagem no aprendizado, reduzindo assim a evasão nos cursos.

\section{Materiais e métodos}

Para o trabalho, foram utilizados dados do Portal do Aluno (https://portal.ufrj.br), disponibilizados pelo Serviço de Informação ao Cidadão (https://ufrj.br/eSIC). Esses dados foram obtidos em 2019, relativos ao ano de 1999 ao de 2018. No entanto, dados sobre características e desempenhos dos alunos foram disponibilizados do período de 2011 a 2018 e são referentes às situações dos alunos no início do segundo semestre de 2018. Nesse período, foram observados 302 alunos nas Física e 173 alunos na Física Médica.

As variáveis selecionadas foram:

a) Curso: Física (F) e Física Médica (FM);

b) Sexo (o Portal do Aluno não especifica gênero);

c) Cor: branco, parto, preto e outros (amarelo ou índio);

a) Categoria de entrada:

- ampla concorrência (A),

- cota racial e por renda (B),

- cota racial independentemente da renda (C),

- cota por renda independentemente da cor (D),

- cota independentemente da cor e da renda (E).
Cabe esclarecer que, antes da Lei das Cotas, só existiam duas categorias de entrada (A e D) e que as cotas se destinam a alunos de escolas públicas.

b) Coeficiente de Rendimento Acumulado (CRA);

c) Média aritmética das notas das cinco áreas de conhecimento do ENEM (0 a 1000);

d) Situação da matrícula: ativa, cancelada ou curso concluído; o cancelamento da matrícula pode ser devido ao jubilamento, mudança de curso, abandono ou trancamento;

e) Motivo de cancelamento da matrícula: abandono, opção de curso, trancamento e jubilamento.

f) Notas nas disciplinas Física I, Física II, Cálculo I e Cálculo II multiplicadas por 10 (0 a 100).

As análises foram realizadas pelo pacote estatístico $R$ versão 3.4.4 [7]. Para a comparação entre dois grupos, utilizou-se o teste t de Student. Para medir associação entre variáveis, foi utilizado modelo de regressão linear. Foram também calculados intervalos de confiança (IC) de $95 \%$ [8] e utilizados gráficos de barra.

\section{Resultados}

\subsection{Evolução temporal dos cursos de 1999 a 2018}

Desde 1999, 1141 alunos se matricularam no curso de Física e 306 alunos no curso de Física Médica.

A evolução percentual anual do número de alunos que se matriculam em cada curso revela um crescimento da Física Médica no IF, sendo de 4,5\% em 1999 e 41,1\% em 2018 (Figura 1).

Desde 2011, ano da separação dos bacharelados, a Física Médica se consolidou com uma média de 23,6 alunos inscritos por ano. Em 2018, o número de vagas ofertadas aumentou de 20 para 30.

No primeiro semestre de 2019, os alunos com matrículas ativas mais antigas foram de 2011 na Física e de 2013 na Física Médica. Dessa forma, a média percentual de desistência nos anos em que não há mais alunos ativos (1999 a 2010) foi de 75,5\% na Física e de 46,7\% na Física Médica (Figura 2).

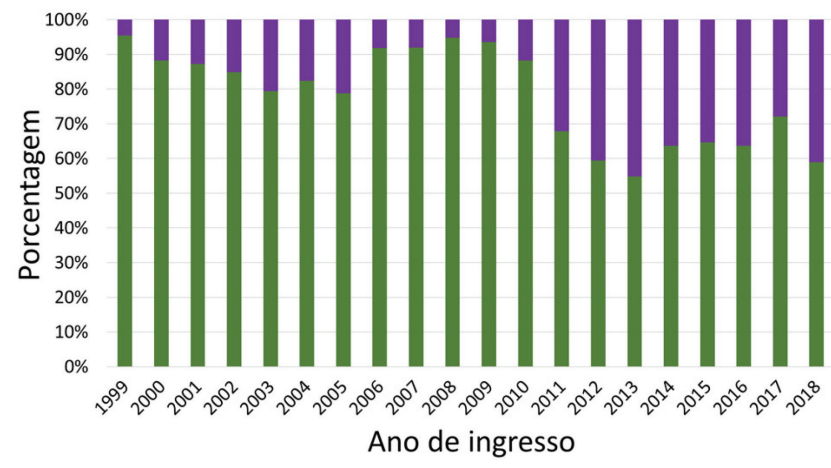

Figura 1: Porcentagem do número total de alunos (Física + Física Médica) por ano de ingresso em cada curso. Física em verde e Física Médica em lilás. 


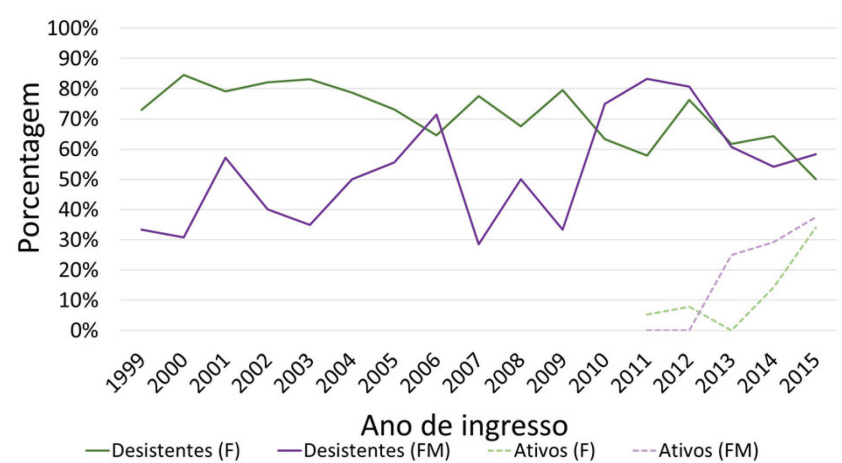

Figura 2: Porcentagem de desistentes e ativos na Física (F) e na Física Médica (FM). A curva em lilás representa os alunos de Física Médica enquanto que a curva em verde os alunos de Física. As curvas pontilhadas correspondem aos alunos ativos.

\subsection{Características dos alunos que ingressaram entre 2011 e 2018}

\subsubsection{Sexo}

Ao longo dos anos, a Física apresentou um percentual médio anual de $25,5 \%$ de alunas do sexo feminino, com exceção do ano de 2018 que teve um percentual de 40,0\%. Na Física Médica, o percentual médio foi de $52,4 \%$ (Figura 3).

\subsubsection{Categoria de entrada}

A porcentagem de cotistas cresceu devido à Lei das Cotas em 2014 e permaneceu em torno de 50,0\% nos dois cursos. A única diferença entre os cursos encontra-se nas porcentagens de alunos de cada tipo de cota devido ao preenchimento das vagas ociosas, principalmente no ano de 2018 na Física Médica (Figura 4). As porcentagens de alunos de cada categoria de entrada nos dois cursos são descritas na Tabela 1

\subsubsection{Cor}

No período analisado, foi observado um percentual médio de $30,8 \%$ de alunos pretos e pardos nos dois cur-

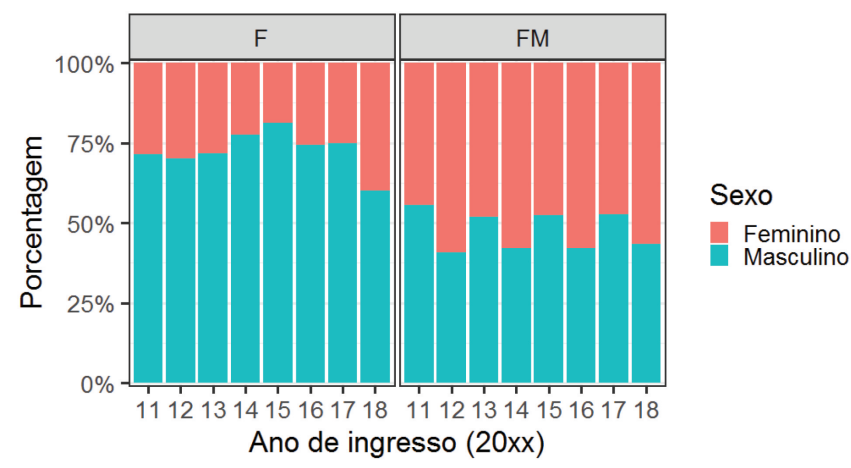

Figura 3: Porcentagem de sexo por ano de ingresso em cada curso.

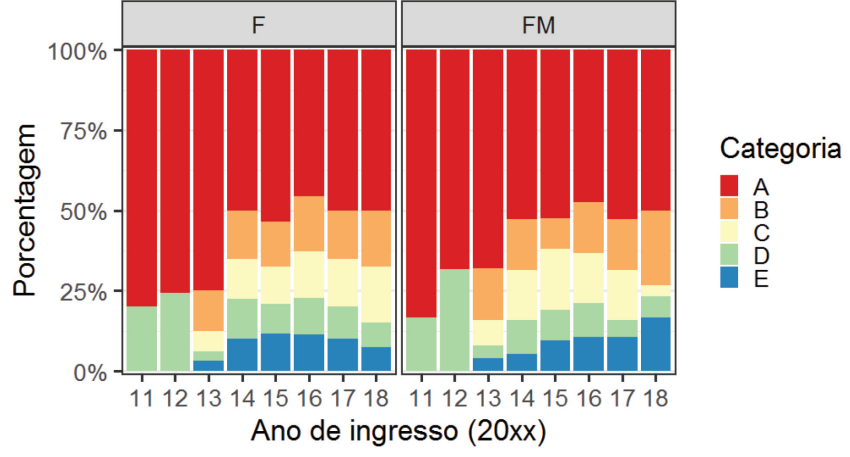

Figura 4: Porcentagem de categoria de entrada por ano de ingresso em cada curso: A- ampla concorrência; B- cota racial e por renda; C- cota racial independentemente da renda; D- cota independentemente da cor por renda e E- cota independentemente da cor e da renda.

Tabela 1: Porcentagens de alunos de cada categoria de entrada nos dois cursos.

\begin{tabular}{ccc}
\cline { 2 - 3 } $\mathbf{A}$ & $\mathbf{F}$ & $\mathbf{F M}$ \\
\cline { 2 - 3 } $\mathbf{B}$ & $59,3 \%$ & $59,0 \%$ \\
$\mathbf{B}$ & $11,6 \%$ & $12,7 \%$ \\
$\mathbf{C}$ & $9,9 \%$ & $9,2 \%$ \\
$\mathbf{D}$ & $12,3 \%$ & $11,6 \%$ \\
$\mathbf{E}$ & $7,0 \%$ & $7,5 \%$ \\
\cline { 2 - 3 }
\end{tabular}

sos. Desde a inclusão das cotas raciais em 2013, o percentual de alunos pretos tem crescido nos últimos anos na Física, mas esse fato não foi observado na Física Médica (Figura 5). Do número de pretos e pardos em cada curso, $64,0 \%$ e $65,7 \%$ entraram por cotas raciais na Física e na Física Médica, respectivamente.

As porcentagens de alunos por cor nos dois cursos são descritas na Tabela 2

\subsubsection{Situação da matrícula}

As porcentagens de alunos com situação da matrícula ativa, cancelada e concluída no início do segundo semestre de 2018 estão na Tabela 3.

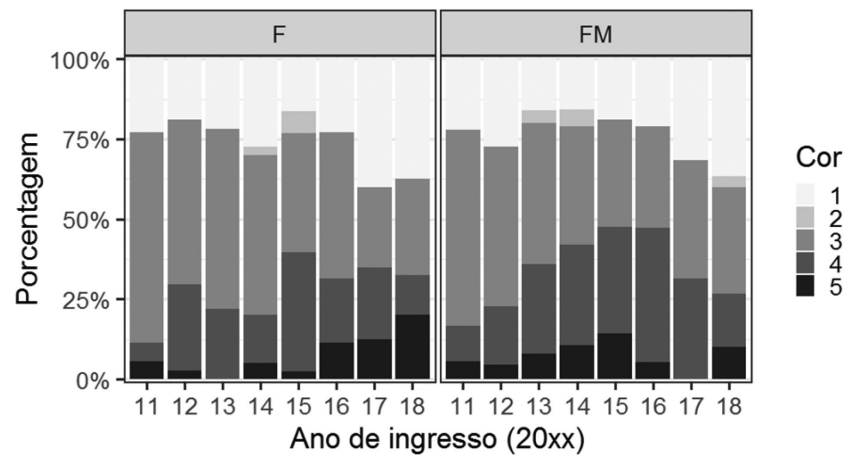

Figura 5: Porcentagem de cor por ano de ingresso em cada curso: 1- Não informado; 2- Outros (amarelo ou índio); 3Branco; 4- Pardo; 5- Preto. 
Tabela 2: Porcentagens de alunos de cada cor nos dois cursos.

\begin{tabular}{lcc}
\cline { 2 - 3 } 1: Não Informado & $\mathbf{F}$ & $\mathbf{F M}$ \\
\cline { 2 - 3 } 2: & $26,2 \%$ & $24,3 \%$ \\
3: Butros & $1,3 \%$ & $1,7 \%$ \\
4: Pardo & $44,4 \%$ & $40,5 \%$ \\
5: Preto & $20,5 \%$ & $26,0 \%$ \\
& $7,6 \%$ & $7,5 \%$ \\
\hline
\end{tabular}

Tabela 3: Porcentagem da situação da matrícula em cada curso.

\begin{tabular}{ccc}
\cline { 2 - 3 } Ativa & $\mathbf{F}$ & $\mathbf{F M}$ \\
\cline { 2 - 3 } Cancelada & $43,4 \%$ & $44,5 \%$ \\
Conclusão & $12,2 \%$ & $49,1 \%$ \\
\cline { 2 - 3 } & & $6,4 \%$ \\
\hline
\end{tabular}

Tabela 4: Porcentagem do motivo de desistência em cada curso.

\begin{tabular}{ccc}
\cline { 2 - 3 } Abandono & $\mathbf{F}$ & $\mathbf{F M}$ \\
\cline { 2 - 3 } Opção de curso & $57,5 \%$ & $56,5 \%$ \\
Jubilamento & $1,5 \%$ & $18,8 \%$ \\
Trancamento & $20,1 \%$ & $17,7 \%$ \\
\cline { 2 - 3 }
\end{tabular}

\subsubsection{Motivo de cancelamento da matrícula}

Na Tabela 4. são apresentadas as porcentagens do motivo de desistência em relação aos alunos com matrícula cancelada em cada curso.

\subsection{ENEM e CRA}

A média do ENEM para entrada dos alunos no curso de Física foi de 694,0 e para a Física Médica foi de 634,2. Essas médias possuem diferença significativa com valor $\mathrm{p}<2,20 \mathrm{e}-16$.

A média do CRA de todos os alunos de Física foi de 4,95 e para a Física Médica de 3,50, sendo observada diferença significativa com valor $\mathrm{p}=1,61 \mathrm{e}-8$. Para os alunos ativos, observou-se diferença (valor $\mathrm{p}=7,47 \mathrm{e}-8$ ), porém, a diferença não foi significativa para os alunos concluintes (valor $\mathrm{p}=0,38$ ).

A associação entre as variáveis ENEM x CRA foi avaliada em um modelo de regressão linear, no qual o $\mathrm{R}^{2}$ foi de 0,238 para Física e de 0,143 para Física Médica.

A seguir é apresentada a descrição das comparações realizadas entre os cursos para as variáveis: categoria de entrada, sexo, cor, situação e cancelamento de matrícula. Na Tabela S1 do Suplemento, são apresentados os valores de média e intervalo de confiança dos escores do ENEM e do CRA de cada curso por categoria de variável.

\section{(a) Por categoria de entrada}

Em relação à nota no ENEM, foram observadas diferenças estatísticas significativas entre os cursos em todas as categorias de entrada. Em relação ao CRA, as médias dos alunos nas categorias B, C e D não apresentaram diferenças estatísticas significativas.

\section{(b) Por sexo}

Em relação à nota no ENEM e ao CRA, foram observadas diferenças estatísticas significativas entre os cursos para as categorias da variável sexo. Cabe destacar que não ocorreram diferenças significativas nas notas dos alunos por sexo em cada curso.

\section{(c) Por Cor}

Em relação à nota no ENEM, diferenças estatísticas significativas não foram observadas para as médias das categorias 2 (outros) e 5 (pretos). O mesmo ocorreu nas médias de CRA, mas com a adição da categoria 3 (pardos).

\section{(d) Por situação da matrícula}

Em relação à nota do ENEM e ao CRA, os alunos com matrículas ativas e canceladas possuem diferenças significativas. No entanto, para os alunos concluintes, não foram observadas diferenças significativas.

(e) Por cancelamento de matrícula

Em relação ao ENEM, os alunos que cancelaram a matrícula de Física Médica tiveram médias menores do que os alunos que cancelaram a matrícula no curso nas situações de: abandono, mudança de curso e trancamento. No entanto, essas diferenças não ocorreram para o CRA.

\subsection{Notas nas disciplinas do ciclo básico}

As médias das notas dos alunos em Cálculo I foram diferentes na comparação entre os cursos (valor $\mathrm{p}<2,20 \mathrm{e}-$ 16), sendo de 3,84 para os alunos de Física e de 1,96 para os alunos de Física Médica. Essa diferença diminuiu em Cálculo II (valor $\mathrm{p}=0,03$ ), cujas médias foram de 4,38 e 3,69 , respectivamente.

Em Física I, as médias na comparação entre os cursos também foram diferentes (valor $\mathrm{p}<2,20 \mathrm{e}-16$ ), sendo de 3,74 para os alunos de Física e de 2,10 para os alunos de Física Médica. Essa diferença diminuiu em Física II (valor $\mathrm{p}=0,001$ ), onde as médias foram de 4,09 e 3,21, respectivamente.

Comparando as médias das notas dos alunos de cada curso ao longo dos anos em Cálculo II e Física II, não foram observadas diferenças significativas. A Figura S1 do Suplemento mostra graficamente os valores de médias e intervalos de confiança no período de 2011 a 2018 por disciplina.

\section{Discussões e conclusões}

\subsection{Quanto ao ingresso}

- A média percentual de desistência nos anos em que não há mais alunos ativos (1999 a 2010) foi maior na Física que na Física Médica. 
- A distribuição entre os sexos é desigual na Física e equilibrada na Física Médica.

- O número de pretos aumentou na Física depois da adesão das cotas raciais em 2013.

- A média das notas no ENEM dos alunos de Física Médica foi ligeiramente menor que a médias dos alunos de Física. A mesma diferença ocorreu ao verificar os alunos em subgrupos como sexo e categoria de entrada. Em relação à cor, só não foram constatadas diferenças entre as médias dos alunos pretos e outros (amarelos ou índios).

- Em cada curso, as médias das notas dos cotistas no ENEM foram menores que as dos alunos que entraram por ampla concorrência, exceto os cotistas E (cota independentemente da cor e da renda) na Física.

- Na Física, a média dos alunos de cor branca foi maior que as médias dos alunos de outras cores, incluindo dos alunos que não informaram a cor. $\mathrm{Na}$ Física Médica, essa diferença não foi observada.

\subsection{Quanto ao desempenho}

- Há diferença no desempenho acadêmico dos alunos de Física e de Física Médica. Essa diferença também foi verificada na comparação dos subgrupos como sexo e cor, com exceção dos alunos de cor branca e não informada. No subgrupo categoria de entrada, foram verificadas diferenças no rendimento acadêmico dos alunos que entraram pela ampla concorrência e pela cota E (cota independentemente da cor e da renda).

- Em cada curso, sexo não influencia o desempenho acadêmico. Ademais, a média dos alunos de cor branca não apresentou diferença significativa em relação aos alunos de cor preta, porém, apresentou em relação aos alunos de cor parda e não informada. Em relação à categoria de entrada, os alunos que entraram pela ampla concorrência apresentaram médias maiores que os alunos cotistas, exceto dos cotistas E (cota independentemente da cor e da renda) na Física.

- Os concluintes de ambos os cursos apresentaram desempenhos semelhantes.

- Comparando os cursos, não foram constatadas diferenças nos rendimentos acadêmicos em função do motivo de cancelamento da matrícula. Na Física, a média dos alunos que abandonaram o curso foi menor do que a dos alunos que optaram por mudança de curso. Na Física Médica, não foi observada essa diferença.
- Cálculo I e Física I são as disciplinas nas quais os alunos de Física Médica apresentaram desempenhos mais baixos, o que pode representar barreira para a realização do curso ou motivo para a evasão. Os alunos que superam essas barreiras parecem recuperar-se em Cálculo II e Física II.

- Como não havia nenhum aluno cotista do curso de Física Médica formado no início do segundo semestre de 2018, não foi possível verificar a influência das cotas nos concluintes.

\subsection{Sugestões para trabalhos futuros}

- Seria interessante verificar em enquetes qualitativas se a diferença de desempenho entre os alunos de Física e Física Médica poderia ser devida à diferença de expectativas sobre o curso.

- É necessário um estudo específico sobre o desempenho dos alunos com cota independentemente da cor e da renda (E) para entender esse desempenho diferenciado.

- Seria interessante avaliar o impacto das iniciativas adotadas para melhorar o desempenho dos alunos (por exemplo a disciplina Tópicos de Física), como também incentivar a criação de Tópicos de Cálculo como apoio à disciplina de Cálculo I.

- É importante avaliar a influência da coordenação e do professor sobre o desempenho dos alunos, em especial nas disciplinas básicas.

- É fundamental que o Sistema Integrado de Gestão Acadêmica (SIGA) disponibilize os dados dos alunos, bem como uma ferramenta de análise desses dados. Isso permitiria apoio ao processo de decisão dos responsáveis pelos cursos no sentido de melhorar o desempenho do curso e atender a necessidades dos diferentes perfis do aluno.

- Finalmente, essas análises poderiam ser realizadas em outros cursos da universidade de forma a viabilizar indicadores e gestão acadêmica dos cursos.

\section{Agradecimentos}

Ao Portal do Aluno da UFRJ por possibilitar acesso aos dados do trabalho.

\section{Material suplementar}

Este suplemento apresenta os resultados (valores de média e intervalo de confiança) das análises comparativas entre os cursos para variáveis descritoras dos alunos e para as disciplinas básicas dos cursos. 
Tabela S1: Médias e Intervalos de Confiança (IC) dos escores do ENEM e do CRA da Física e da Física Médica. Em cor vermelha estão destacadas as diferenças significativas entre os cursos por categoria das variáveis.

\begin{tabular}{|c|c|c|c|c|c|}
\hline \multirow[b]{2}{*}{ Variável } & \multirow[b]{2}{*}{ Categoria } & \multicolumn{2}{|c|}{ ENEM } & \multicolumn{2}{|c|}{ CRA } \\
\hline & & $\mathbf{F}$ & FM & $\mathbf{F}$ & FM \\
\hline \multirow[t]{5}{*}{ Cor } & Branco & $707,7[697,7 ; 717,7]$ & $644,7[630,7 ; 658 ; 7]$ & $5,62[5,22 ; 6,03]$ & $4,27[3,67 ; 4,87]$ \\
\hline & Preto & $653,2[629,3 ; 677,1]$ & $613,2[586,7 ; 639,7]$ & $4,60[3,51 ; 5,69]$ & $3,43[2,22 ; 4,64]$ \\
\hline & Pardo & $672,5[660,0 ; 685,1]$ & $618,4[603,1 ; 633,8]$ & $4,29[3,57 ; 5,01]$ & $2,91[2,23 ; 3,59]$ \\
\hline & Outros & $682,1^{*}$ & $671,7^{*}$ & $6,00^{*}$ & $5,35^{*}$ \\
\hline & Não Informado & $703,5[691,1 ; 715,8]$ & $640,0[615,4 ; 664,7]$ & $4,37[3,62 ; 5,12]$ & $2,74[1,91 ; 3,56]$ \\
\hline \multirow[t]{2}{*}{$\overline{\text { Sexo }}$} & Feminino & $688,4[675,6 ; 701,2]$ & $624,6[611,5 ; 637,6]$ & $4,85[4,28 ; 5,42]$ & $3,62[3.11 ; 4,13]$ \\
\hline & Masculino & $696,1[688,4 ; 703,8]$ & $645,6[632,5 ; 658,7]$ & $4,98[4,60 ; 5,37]$ & $3,36[2,79 ; 3,94]$ \\
\hline \multirow{5}{*}{$\begin{array}{l}\text { Categoria de } \\
\text { entrada }\end{array}$} & $\mathrm{A}$ & $718,8[711,4 ; 726,2]$ & $659,7[649,3 ; 670,2]$ & $5,54[5,15 ; 5,93]$ & $4,12[3,62 ; 4,63]$ \\
\hline & B & $644,8[633,7 ; 655,8]$ & $584,1[555,9 ; 612,4]$ & $3,37[2,45 ; 4,96]$ & $2,88[2,05 ; 3,70]$ \\
\hline & $\mathrm{C}$ & $655,1[638,8 ; 671,3]$ & $610,7[589,5 ; 632,0]$ & $3,75[2,68 ; 4,81]$ & $2,65[1,38 ; 3,91]$ \\
\hline & $\mathrm{D}$ & $659,8[640,0 ; 679,1]$ & $613,6[586,7 ; 640,6]$ & $3,82[3,04 ; 4,60]$ & $2,37[1,18 ; 3,56]$ \\
\hline & $\mathrm{E}$ & $702,3[680,8 ; 723,8]$ & $605,4[573,6 ; 636,6]$ & $6,09[4,86 ; 7,32]$ & $2,42[1,08 ; 3,75]$ \\
\hline \multirow{3}{*}{$\begin{array}{l}\text { Situação da } \\
\text { Matrícula }\end{array}$} & Ativa & $703,4[694,0 ; 712,9]$ & $638,1[626,7 ; 649,4]$ & $6,18[5,80 ; 6,55]$ & $4,71[4,31 ; 5,11]$ \\
\hline & Cancelada & $679,8[669,8 ; 689,7]$ & $625,8[610,0 ; 641,7]$ & $3,10[2,66 ; 3,54]$ & $1,94[1,47 ; 2,41]$ \\
\hline & Concluída & $712,9[693,3 ; 732,5]$ & $673,0[630,9 ; 715,1]$ & $7,29[7,00 ; 7,59]$ & $7,07[6,61 ; 7,53]$ \\
\hline \multirow{4}{*}{$\begin{array}{l}\text { Motivo de } \\
\text { cancela- } \\
\text { mento }\end{array}$} & Abandono & $675,2[661,2 ; 689,1]$ & $632,7[607,8 ; 657,5]$ & $2,57[2,03 ; 3,11]$ & $1,90[1,23 ; 2,59]$ \\
\hline & Mudança de curso & $685,9[668,0 ; 703,9]$ & $626,0[597,8 ; 654,1]$ & $4,44[3,43 ; 5,45]$ & $2,43[1,12 ; 3,76]$ \\
\hline & Jubilamento & $625,3^{*}$ & $607,1[564,6 ; 649,6]$ & $2,36^{*}$ & $2,00[0,57 ; 3,43]$ \\
\hline & Trancamento & $688,2[663,8 ; 712,6]$ & $616,8[575,7 ; 657,9]$ & $3,28[2,18 ; 4,38]$ & $1,50[0,69 ; 2,31]$ \\
\hline
\end{tabular}

*Grupos com menos de 5 alunos estão apenas com os valores das médias.
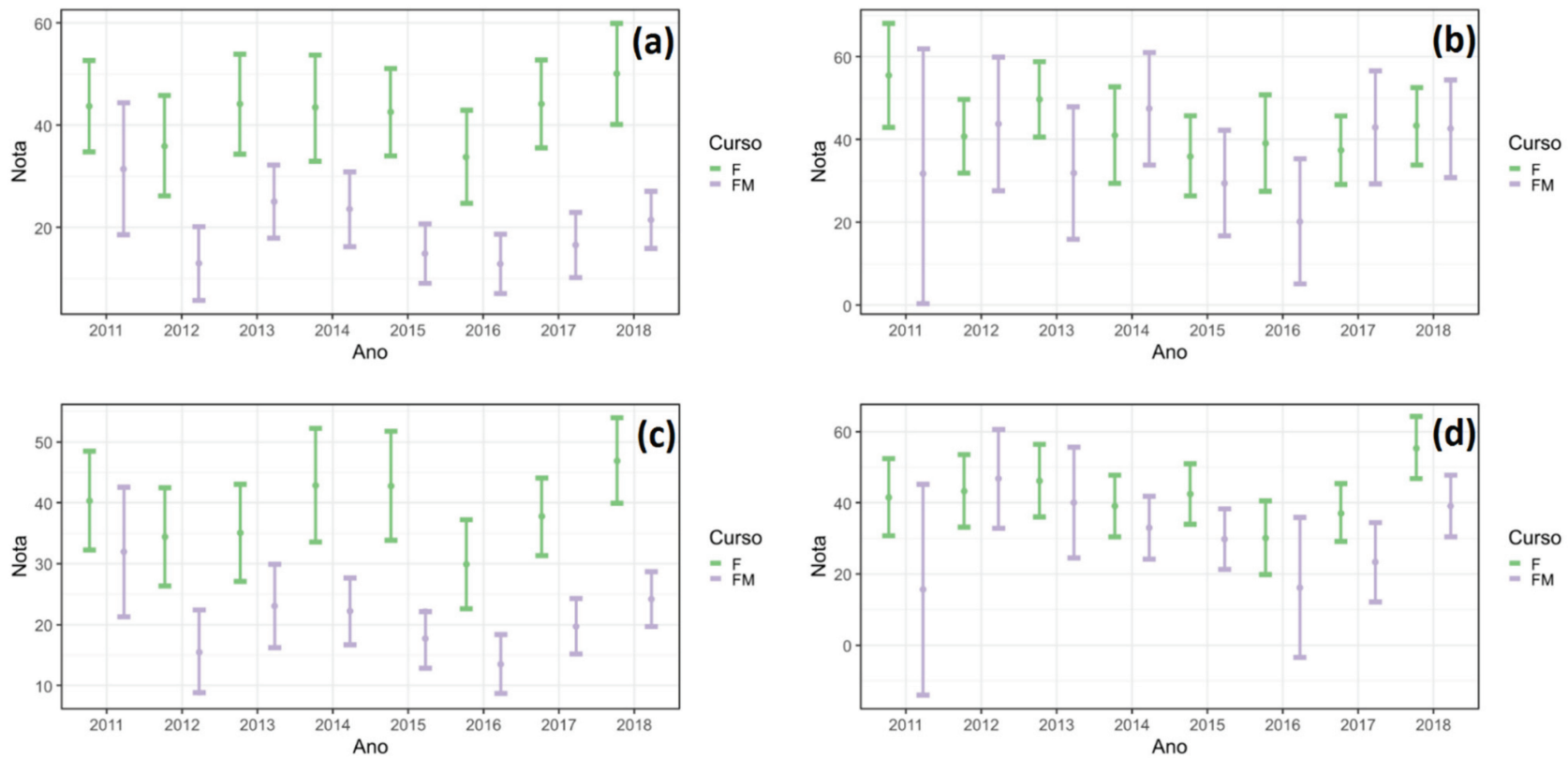

Figura S1: Médias e intervalos de confiança das notas de Cálculo I (a), de Cálculo II (b), de Física I (c) e de Física II (d) em cada curso ao longo dos anos. 


\section{Referências}

[1] COMISSÃO NACIONAL DE ENERGIA NUCLEAR (CNEN). CNEN NN 6.10: Requisitos de segurança e proteção radiológica para serviços de radioterapia. Brasil, 2014. Disponível em: http:// appasp.cnen.gov.br/seguranca/normas/pdf/Nrm610.pdf

[2] COMISSÃO NACIONAL DE ENERGIA NUCLEAR (CNEN). CNEN NN 3.05: Requisitos de segurança e proteção radiológica para serviços de medicina nuclear. Brasil, 2013. Disponível em: http://appasp. cnen.gov.br/seguranca/normas/pdf/Nrm305.pdf

[3] AGÊNCIA NACIONAL DE VIGILÂNCIA SANITÁRIA (ANVISA). Portaria 453, de 01 de junho de 1998. Diretrizes Básicas de Proteção Radiológica em Radiodiagnóstico Médico e Odontológico. Diário Oficial da União, 1998.

[4] AGÊNCIA NACIONAL DE VIGILÂNCIA SANITÁRIA (ANVISA). Resolução da Diretoria Colegiada RDC $\mathrm{n}^{\circ}$ 330, de 20 de Dezembro de 2019. Diário Oficial da União, 2019 .
[5] Universidade Federal do Rio de Janeiro, Sistema Integrado de Gestão Acadêmico - 2020, disponível em: https://siga.ufrj.br/sira/repositorio-curriculo/ListaCurs os.html, acessado em: 17/01/2020.

[6] Universidade Federal do Rio de Janeiro, Edital $n^{\circ} 335$ de 09 de outubro de 2013, disponível em: https://acessograduacao.ufrj.br/processos/2014-1/acesso -2014/divulgacao-dos-editais-para-o-acesso-2014/2014-E dital_335-2013-Acesso_SiSU.pdf acessado em 17/01/ 2020.

[7] R. Core Team (2018). A language and environment for statistical computing. $R$ Foundation for Statistical Computing, Vienna, Austria, disponível em: https://www.rproject.org/

[8] FREIRE, S. M. Bioestatística Básica, disponível em: http://www.lampada.uerj.br/arquivosdb/_book/bioestat isticaBasica.html, acessado em: 17/01/2020. 